\title{
INVARIANT SUBSPACES OF THE MONODROMY
}

\author{
David B. MAssey
}

\begin{abstract}
We show that there are obstructions to the existence of certain types of invariant subspaces of the Milnor monodromy; this places restrictions on the cohomology of Milnor fibres of non-isolated hypersurface singularities.
\end{abstract}

\section{$\S 0$. Introduction}

Let $\mathcal{U}$ be an open neighborhood of the origin in $\mathbb{C}^{n+1}$, where $n \geqslant 2$. Let $f:(\mathcal{U}, \mathbf{0}) \rightarrow(\mathbb{C}, 0)$ be a complex analytic function which has a smooth 1-dimensional critical locus, $\Sigma f$, at the origin. Note that this, combined with the fact that $n \geqslant 2$, implies that $f$ is reduced at the origin.

For $\mathbf{p} \in V(f)$, we are interested in the cohomology of the Milnor fibre $F_{f, \mathbf{p}}$. For technical reasons, it is easier to work with field coefficients, rather than with integer coefficients. Of course, by varying the base field through finite fields, one may still detect torsion in the integral cohomology. Hence, throughout this paper, we fix a field $\mathfrak{K}$, and all cohomology spaces will be $\mathfrak{K}$-vector spaces.

Since we care only about the analytic-type of the germ of $f$ at the origin, we may make an analytic change of coordinates and assume that $\Sigma f$ is, in fact, a portion of the complex line $\mathbb{C} \times\{\mathbf{0}\}$. Thus, we shall assume that $S:=\Sigma f=(\mathbb{C} \times\{\mathbf{0}\}) \cap \mathcal{U}$. In addition, we shall assume that $\mathcal{U}$ has been chosen small enough so that $S \subseteq V(f)$, and so that $\{\mathcal{U}-V(f), V(f)-S, S-\{\mathbf{0}\},\{\mathbf{0}\}\}$ is a Whitney stratification. Finally, let us use $z_{0}$ for the first coordinate of $\mathbb{C}^{n+1}$, and assume that $\mathcal{U}$ is small enough and that $z_{0}$ is generic enough so that $\Sigma\left(f_{\left.\left.\right|_{V\left(z_{0}\right)}\right)}\right)=\{\mathbf{0}\}$.

At each point $\mathbf{p} \in V(f)-S$, the Milnor fibre $F_{f, \mathbf{p}}$ is contractible; hence, at such points, the reduced cohomology $\widetilde{H}^{*}\left(F_{f, \mathbf{p}}\right)=0$.

At each point $\mathbf{p} \in S-\{\mathbf{0}\}$, the Milnor fibre is the cross-product of a disk and the Milnor fibre of a generic hyperplane slice. Hence, at such points, $\widetilde{H}^{*}\left(F_{f, \mathbf{p}}\right)=0$, except in degree $n-1$; we shall denote the dimension of $\widetilde{H}^{n-1}\left(F_{f, \mathbf{p}}\right)$ by $\lambda_{f}^{1}$. (This is the 1-dimensional Lê number; see [M2].) Finally, $\widetilde{H}^{*}\left(F_{f, \mathbf{0}}\right)$ is possibly non-zero only in degrees $n-1$ and $n$.

However, there are further restrictions on the cohomologies of the various $F_{f, \mathbf{p}}$. The cohomology groups $\widetilde{H}^{n-1}\left(F_{f, \mathrm{p}}\right)$ actually form a local system over the punctured disk $S-\{\mathbf{0}\}$; this local system is characterized by fixing a point $\mathbf{p}_{0} \in S-\{\mathbf{0}\}$ and considering the monodromy isomorphism

$$
\nu: \widetilde{H}^{n-1}\left(F_{f, \mathbf{p}_{0}}\right) \rightarrow \widetilde{H}^{n-1}\left(F_{f, \mathbf{p}_{0}}\right),
$$

which is obtained by letting $\mathbf{p}_{0}$ travel around the origin in $S$. We refer to this as the internal monodromy (it is sometimes referred to as the vertical monodromy). Note that this is most definitely not the Milnor monodromy, which results from letting the value of $f$ travel around the origin.

1991 Mathematics Subject Classification. 32B15, 32C35, 32C18, 32B10.

Key words and phrases. Milnor fibre, vanishing cycles, monodromy, perverse sheaves. 
Even at the origin, the cohomology of the Milnor fibre is not independent of the local system determined by $\nu$. The relationships between the cohomology of $F_{f, \mathbf{0}}$ and the cohomologies of the other $F_{f, \mathbf{p}}$ are encoded by the complex of sheaves of vanishing cycles, $\phi_{f} \mathfrak{K}_{\mathcal{u}}^{\bullet}$ (see $[\mathbf{D}]$, [K-S]); this complex of sheaves is defined on all of $V(f)$, but is supported on $S$. It is more convenient to restrict the vanishing cycles to their support $S$ and, for technical reasons, to shift the complex into non-positive degrees; hence, we let $\mathbf{P}^{\bullet}:=\left(\phi_{f} \mathfrak{K}_{\mathfrak{u}}^{\bullet}\right)_{\mid S}[n]$.

Can we obtain, up to isomorphism, every bounded constructible complex of $\mathfrak{K}$-vector spaces on a complex line by varying the function $f$ through all analytic functions with line singularities and looking at the corresponding $\mathbf{P}^{\bullet}$ 's? Certainly not. The discussion above indicates restrictions on the degrees in which one may have non-zero cohomology; after our shift, we find that $\mathbf{P}_{\left.\right|_{S-\{\mathbf{0}}}$ is a local system, concentrated in degree -1 and characterized by $\nu$, while the stalk $\mathbf{P}_{\mathbf{0}}^{\bullet}$ has possibly non-zero cohomology only in degrees -1 and 0 . All of these properties are a reflection of the well-known fact that $\mathbf{P}^{\bullet}$ must be a perverse sheaf on $S$.

In fact, the properties above almost completely describe perverse sheaves on $S$, which are constructible with respect to $\{S-\{\mathbf{0}\},\{\mathbf{0}\}\}$. The only additional required property is implied by the cosupport condition at the origin: the inclusion map $i: S-\{\mathbf{0}\} \hookrightarrow S$ must induce an injection

$$
H^{-1}\left(\mathbf{P}^{\bullet}\right)_{\mathbf{0}} \cong \mathbb{H}^{-1}\left(S ; \mathbf{P}^{\bullet}\right) \hookrightarrow \mathbb{H}^{-1}\left(S-\{\mathbf{0}\} ; \mathbf{P}^{\bullet}\right) .
$$

Using that the hypercohomology of a local system (in degree -1) on a punctured disk is given by $\mathbb{H}^{-1}\left(S-\{\mathbf{0}\} ; \mathbf{P}^{\bullet}\right) \cong \operatorname{ker}(\mathrm{id}-\nu)\left(\right.$ and $\left.\mathbb{H}^{0}\left(S-\{\mathbf{0}\} ; \mathbf{P}^{\bullet}\right) \cong \operatorname{coker}(\mathrm{id}-\nu)\right)$, this extra condition can be stated as: $i$ induces an inclusion

$$
\widetilde{H}^{n-1}\left(F_{f, 0}\right) \hookrightarrow \operatorname{ker}(\mathrm{id}-\nu) .
$$

The reasonable question to ask now is: can every perverse sheaf on $S$ be obtained by varying the function $f$ ? Again, the answer is "no"; the complex $\mathbf{P}^{\bullet}$ must be self-dual, i.e., $\mathbf{P}^{\bullet}$ must be isomorphic to its own Verdier dual; this is well-known (it follows from non-natural isomorphisms $\mathcal{D} \circ \phi_{f}[-1] \cong \phi_{f}[-1] \circ \mathcal{D}$ and $\mathcal{D}\left(\mathfrak{K}_{\mathcal{U}}^{\bullet}[n+1]\right) \cong \mathfrak{K}_{\mathcal{U}}^{\bullet}[n+1]$; see, for instance, [B] $\left.]\right)$. In Section 1, we shall use MacPherson and Vilonen's results in $[\mathbf{M}-\mathbf{V}]$ to provide a description of the category of perverse sheaves on a line, and then we shall be able to explain self-duality in a down-to-Earth manner. MacPherson and Vilonen's description is essential for the understanding the results of this paper.

The goal of this paper is to show that there are further general restrictions on the vanishing complex $\mathbf{P}^{\bullet}$, i.e., one can not even obtain every self-dual perverse sheaf on $S$ by varying $f$; there are invariant subspaces of the monodromy isomorphism which impose additional structure on $\mathbf{P}^{\bullet}$. This result implies new restrictions on the cohomology of the Milnor fibre of a line singularity in affine space.

However, despite the fact that our methods use the derived category and perverse sheaves, the statement of our main theorem is very classical.

Given the complex analytic function $f:(\mathcal{U}, \mathbf{0}) \rightarrow(\mathbb{C}, 0)$, whose critical locus at the origin is a line, we may select a generic linear form $L$, and consider the one-parameter family of isolated singularities given by $f_{t}:=f_{\left.\right|_{V(L-L(t))}}$, and we may consider the corresponding Milnor numbers $\mu_{t}$. For all small $t \neq 0$, the $\mu_{t}$ have a common value, namely $\lambda_{f}^{1}$. The main theorem of this paper is Theorem 3.3:

Theorem. If $\mu_{0}=1+\lambda_{f}^{1}$, then there is a strict inequality $\operatorname{dim}_{\mathfrak{K}} \widetilde{H}^{n-1}\left(F_{f, \mathbf{0}}\right)<\lambda_{f}^{1}$.

The previously-known general bound on the reduced cohomology in degree $n-1$ was the non-strict inequality $\operatorname{dim}_{\mathfrak{K}} \widetilde{H}^{n-1}\left(F_{f, \mathbf{o}}\right) \leqslant \lambda_{f}^{1}$. 


\section{$\S 1$. The Category of Perverse Sheaves on a Line}

We continue with the notation from the introduction.

In order to explain what self-duality implies, it will be useful to avail ourselves of the description of the category of perverse sheaves on a line which is provided by MacPherson and Vilonen in [M-V].

If $g: \mathcal{U} \rightarrow \mathbb{C}$ is any complex analytic function, we may consider the functors $\psi_{g}[-1]$ and $\phi_{g}[-1]$ given by shifting the nearby and vanishing cycles, respectively, i.e., for a complex $\mathbf{F}^{\bullet}$ on $\mathcal{U}, \psi_{g}[-1] \mathbf{F}^{\bullet}:=$ $\left(\psi_{g} \mathbf{F}^{\bullet}\right)[-1]$ and $\phi_{g}[-1] \mathbf{F}^{\bullet}:=\left(\phi_{g} \mathbf{F}^{\bullet}\right)[-1]$. The reason for defining these shifted functors is that $\psi_{g}[-1]$ and $\phi_{g}[-1]$ take perverse sheaves to perverse sheaves, i.e., they yield functors from the Abelian category of perverse sheaves on $\mathcal{U}$ to the Abelian category of perverse sheaves on $V(g)$.

Now, suppose that $\mathbf{Q}^{\bullet}$ is an arbitrary perverse sheaf on the line $S$, and let $\hat{z}_{0}:=\left.z_{0}\right|_{S}$. Then, $\phi_{\hat{z}_{0}}[-1] \mathbf{Q}^{\bullet}$ and $\psi_{\hat{z}_{0}}[-1] \mathbf{Q}^{\bullet}$ are perverse sheaves on the single point $\mathbf{0}$, and consequently have possibly non-zero cohomology only in degree 0 . Let $W:=H^{0}\left(\phi_{\hat{z}_{0}}[-1] \mathbf{Q}^{\bullet}\right)_{0}$ and $V:=H^{0}\left(\psi_{\hat{z}_{0}}[-1] \mathbf{Q}^{\bullet}\right)_{0}$. Then, there is the canonical map $\gamma: V \rightarrow W$ and the variation map $\delta: W \rightarrow V$, and $\delta \circ \gamma=$ id $-\nu$, where $\nu$ is, again, the internal monodromy isomorphism of $\mathbf{Q}_{\left.\right|_{S-\{\mathbf{0}\}}}$, which arises from considering what happens as the stalks travel around a small circle centered at the origin in $S$.

Thus, a perverse sheaf on $L$ yields two vector spaces, $V$ and $W$, an isomorphism $\nu: V \rightarrow V$, and maps $\gamma: V \rightarrow W$ and $\delta: W \rightarrow V$ such that $\delta \circ \gamma=\mathrm{id}-\nu$. This situation is nicely represented by a commutative triangle

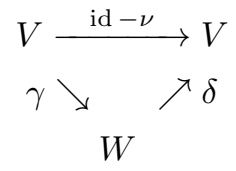

We refer to such a commutative diagram as an $M$ - $V$ triangle. The category of perverse sheaves on $S$ (constructible with respect to $\{S-\{\mathbf{0}\},\{\mathbf{0}\}\}$ ) is equivalent to the category of M-V triangles, where a morphism of M-V triangles is defined in the obvious way: a morphism is determined by linear maps $\tau: V \rightarrow V^{\prime}$ and $\eta: W \rightarrow W^{\prime}$ such that

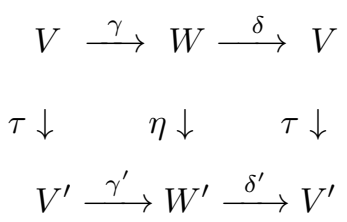

commutes.

Note that one can recover the stalk cohomology of $\mathbf{Q}^{\bullet}$ at the origin from the associated $\mathrm{M}-\mathrm{V}$ triangle:

$$
H^{-1}\left(\mathbf{Q}^{\bullet}\right)_{\mathbf{0}} \cong \operatorname{ker} \gamma \quad \text { and } \quad H^{0}\left(\mathbf{Q}^{\bullet}\right)_{\mathbf{0}} \cong \operatorname{coker} \gamma
$$

We can now describe Verdier dualizing. If a perverse sheaf, $\mathbf{Q}^{\bullet}$, on $S$ has its $\mathrm{M}-\mathrm{V}$ triangle given by

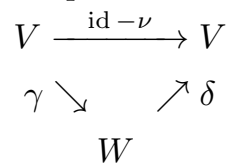

then the M-V triangle of the Verdier dual of $\mathbf{Q}^{\bullet}, \mathcal{D} \mathbf{Q}^{\bullet}$, is isomorphic to the dual triangle 


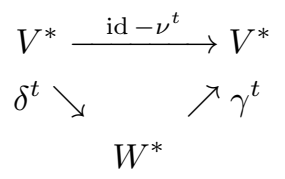

Now, we return to the situation of the introduction, and consider the case where $\mathbf{Q}^{\bullet}=\mathbf{P}^{\bullet}:=$ $\left(\phi_{f} \mathfrak{K}_{\mathcal{u}}^{\bullet}\right)_{\left.\right|_{S}}[n]$. The $\mathrm{M}-\mathrm{V}$ triangle of $\mathbf{P}^{\bullet}$,

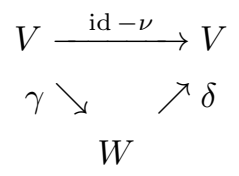

has $V \cong \mathfrak{K}^{\lambda_{f}^{1}}$ and $W \cong \mathfrak{K}^{\lambda_{f}^{0}}$, where $\lambda_{f}^{0}$ is the zero-dimensional Lê number (again, see [M2]). This 0 -dimensional Lê number can calculated effectively; let $\Gamma_{f, z_{0}}^{1}$ denote the relative polar curve, then we have $\lambda_{f}^{0}=\left(\Gamma_{f, L}^{1} \cdot V\left(\frac{d f}{d L}\right)\right)_{0}$. Note that

$$
\operatorname{ker} \gamma \cong \widetilde{H}^{n-1}\left(F_{f, \mathbf{0}}\right) \quad \text { and } \quad \text { coker } \gamma \cong \widetilde{H}^{n}\left(F_{f, \mathbf{o}}\right)
$$

and the self-duality of $\mathbf{P}^{\bullet}=\left(\phi_{f} \mathfrak{K}_{\mathcal{u}}^{\bullet}\right)_{\left.\right|_{S}}[n]$ is equivalent to saying that its M-V triangle is (non-naturally) isomorphic to its dual.

\section{$\S 2$. The Morse Modification}

In this section, we shall place ourselves in a more general setting, and show how the Morse-theoretic result of Lê in $[\mathbf{L}]$ actually yields an important method of modifying the sheaf of vanishing cycles.

Throughout this section, we continue with $\mathcal{U}$ being an open neighborhood of the origin in $\mathbb{C}^{n+1}$, and let $g:(\mathcal{U}, \mathbf{0}) \rightarrow(\mathbb{C}, 0)$ be a complex analytic function (with an arbitrary critical locus). Let $L: \mathbb{C}^{n+1} \rightarrow \mathbb{C}$ be a linear form.

Lê's attaching formula in $[\mathbf{L}]$ provides data as to how the Milnor fibre, $F_{g, \mathbf{0}}$, is built from the Milnor fibre, $F_{g_{\mid V(L)}}, \mathbf{0}$, of the hyperplane slice:

Theorem 2.1 (Lê, $[\mathbf{L}])$. For a generic choice of $L$, the relative cohomology $H^{*}\left(F_{g, \mathbf{0}}, F_{g_{\left.\right|_{V(L)}}, \mathbf{0}} ; \mathbb{Z}\right)$ is zero in all degrees except, possibly, in degree $n$. In addition, $H^{n}\left(F_{g, \mathbf{0}}, F_{g_{\left.\right|_{V(L)}}, \mathbf{0}} ; \mathbb{Z}\right)$ is free Abelian of rank equal to the intersection number of the polar curve $\Gamma_{g, L}^{1}$ and the hypersurface $V(g)$ at the origin. Furthermore, for all $\mathbf{x} \in V(g) \cap V(L)-\{\mathbf{0}\}$ near $\mathbf{0}, H^{*}\left(F_{g, \mathbf{x}}, F_{g_{\mid}(L)}, \mathbf{x} ; \mathbb{Z}\right)$ is zero in all degrees.

In our own paper $[\mathbf{M}]$, we generalize the above result to the case where the coefficients are an arbitrary bounded, constructible complex of sheaves. Applying that result to the special case of perverse sheaves (e.g., the shifted constant sheaf $\mathfrak{K}_{\mathcal{u}}^{\bullet}[n+1]$ ), we obtain:

Theorem 2.2 (Massey, $[\mathbf{M}]$ ). Let $\mathbf{Q}^{\bullet}$ be a perverse sheaf of $\mathfrak{K}$-vector spaces on $\mathcal{U}$. For a generic choice of $L$, the relative hypercohomology $\mathbb{H}^{*}\left(F_{g, \mathbf{0}}, F_{g_{\mid}(L)}, \mathbf{0} ; \mathbf{Q}^{\bullet}\right)$ is zero in all degrees except, possibly, in 
degree 0. Furthermore, for all $\mathbf{x} \in V(g) \cap V(L)-\{\mathbf{0}\}$ near $\mathbf{0}, \mathbb{H}^{*}\left(F_{g, \mathbf{x}}, F_{g_{\left.\right|_{V}(L)}, \mathbf{x}} ; \mathbf{Q}^{\bullet}\right)$ is zero in all degrees.

Remark 2.3. In the situation of Theorem 2.2, the results of $[\mathbf{M}]$ also tell one how to calculate the dimension over $\mathfrak{K}$ of $\mathbb{H}^{n}\left(F_{g, \mathbf{0}}, F_{g_{\left.\right|_{V(L)}}, \mathbf{0}} ; \mathbf{Q}^{\bullet}\right)$. As in Theorem 2.1, the intersection number $\left(\Gamma_{g, L}^{1} \cdot V(g)\right)_{\mathbf{0}}$ appears, but now one must calculate this intersection number for various strata, multiply by contributions from the normal data to strata (the Morse modules of strata), and then take the sum over all strata. We shall not need that calculation in this paper.

We wish to encode the result in Theorem 2.2 in a complex of sheaves.

Let $\mathbf{Q}^{\bullet}$ be a perverse sheaf of $\mathfrak{K}$-vector spaces on $\mathcal{U}$. Fix a linear form $L$. Let $j: \mathcal{U} \cap V(L) \hookrightarrow \mathcal{U}$ denote the closed inclusion, and let $i: \mathcal{U}-\mathcal{U} \cap V(L) \hookrightarrow \mathcal{U}$ denote the open inclusion. In the derived category, there is a fundamental distinguished triangle

$$
j_{*} j^{*} \mathbf{Q}^{\bullet}[-1] \rightarrow i_{!} i \mathbf{Q}^{\bullet} \rightarrow \mathbf{Q}^{\bullet} \stackrel{[1]}{\longrightarrow} j_{*} j^{*} \mathbf{Q}^{\bullet}[-1]
$$

the associated long exact sequence on hypercohomology is the relative hypercohomology long exact sequence of the pair $(\mathcal{U}, \mathcal{U} \cap V(L))$ :

$$
\cdots \rightarrow \mathbb{H}^{i-1}\left(\mathcal{U} \cap V(L) ; \mathbf{Q}^{\bullet}\right) \rightarrow \mathbb{H}^{i}\left(\mathcal{U}, \mathcal{U} \cap V(L) ; \mathbf{Q}^{\bullet}\right) \rightarrow \mathbb{H}^{i}\left(\mathcal{U} ; \mathbf{Q}^{\bullet}\right) \rightarrow \mathbb{H}^{i}\left(\mathcal{U} \cap V(L) ; \mathbf{Q}^{\bullet}\right) \rightarrow \ldots
$$

In the distinguished triangle $(\dagger), \mathbf{Q}^{\bullet}$ and $i_{!} i^{!} \mathbf{Q}^{\bullet}$ are perverse, but $j_{*} j^{*} \mathbf{Q}^{\bullet}[-1]$ need not be. However, in the important case where $\mathbf{Q}^{\bullet}=\mathfrak{K}_{\mathcal{U}}^{\bullet}[n+1]$, it is, in fact, true that $j_{*} j^{*} \mathbf{Q}^{\bullet}[-1] \cong j_{*} \mathfrak{K}_{\mathcal{U} \cap V(L)}^{\bullet}[n]$ is perverse.

There is also a canonical distinguished triangle relating the nearby and vanishing cycles:

$$
\left(i_{!} i \mathbf{Q}^{\bullet}\right)_{\left.\right|_{V(g)}} \rightarrow \psi_{g}[-1]\left(i_{!} i \mathbf{Q}^{\bullet}\right) \rightarrow \phi_{g}[-1]\left(i_{!} i^{!} \mathbf{Q}^{\bullet}\right) \stackrel{[1]}{\longrightarrow}\left(i_{!} i \mathbf{Q}^{\bullet}\right)_{\left.\right|_{V(g)}}
$$

Let $l: V(g) \cap V(L) \hookrightarrow V(g)$ denote the inclusion. Applying the functors $\psi_{g}[-1]$ and $\phi_{g}[-1]$ to the distinguished triangle $(\dagger)$, comparing parts of the resulting distinguished triangles via $(*)$, and applying Theorem 2.2 , we see that, for generic linear forms $L$, the perverse sheaf $\phi_{g}[-1]\left(i_{!} i^{!} \mathbf{Q}^{\bullet}\right)$ has the following four properties:

i) there is a distinguished triangle

$$
l_{*} \phi_{g_{\left.\right|_{V}(L)}}[-1]\left(\mathbf{Q}_{\left.\right|_{V(L)}}^{\bullet}[-1]\right) \rightarrow \phi_{g}[-1]\left(i_{!} i \mathbf{Q}^{\bullet}\right) \stackrel{\alpha}{\rightarrow} \phi_{g}[-1]\left(\mathbf{Q}^{\bullet}\right) \stackrel{[1]}{\rightarrow} l_{*} \phi_{g_{\left.\right|_{V(L)}}}[-1]\left(\mathbf{Q}_{\left.\right|_{V(L)}}^{\bullet}[-1]\right)
$$

ii) if $\mathbf{x} \in V(g)-V(L)$, the morphism $\alpha$ above induces an isomorphism on stalk cohomology

$$
H^{*}\left(\phi_{g}[-1]\left(i_{!} i \mathbf{Q}^{\bullet}\right)\right)_{\mathbf{x}} \cong H^{*}\left(\phi_{g}[-1]\left(\mathbf{Q}^{\bullet}\right)\right)_{\mathbf{x}} ;
$$

iii) if $\mathbf{x} \in V(g) \cap V(L)-\{\mathbf{0}\}$ and $\mathbf{x}$ is near $\mathbf{0}, H^{*}\left(\phi_{g}[-1]\left(i_{!} i^{!} \mathbf{Q}^{\bullet}\right)\right)_{\mathbf{x}}=0$; 
iv) $H^{*}\left(\phi_{g}[-1]\left(i_{i} i \mathbf{Q}^{\bullet}\right)\right)_{\mathbf{0}}$ is zero in all degrees except, possibly, degree 0 .

Remark 2.4. Note that, as $L$ is generic, ii) and iii) imply that $\operatorname{supp} \phi_{g}[-1]\left(i_{!} i^{!} \mathbf{Q}^{\bullet}\right)=\operatorname{supp} \phi_{g}[-1] \mathbf{Q}^{\bullet}$ near $\mathbf{0}$. Note also that, as mentioned in Remark 2.3, it is possible to give a formula for the dimension $\operatorname{dim}_{\mathfrak{K}} H^{0}\left(\phi_{g}[-1]\left(i_{!} i^{!} \mathbf{Q}^{\bullet}\right)\right)_{\mathbf{0}}$ in terms of intersection numbers and Morse modules of strata.

Definition 2.5. We refer to $\phi_{g}[-1]\left(i_{!} i^{!} \mathbf{Q}^{\bullet}\right)$ as the Morse modification of $\phi_{g}[-1]\left(\mathbf{Q}^{\bullet}\right)$, with respect to $L$ at $\mathbf{0}$

We let $\overline{\Sigma_{\mathbf{Q}} \cdot g}:=\operatorname{supp} \phi_{g}[-1] \mathbf{Q}^{\bullet}$, and we refer to the restriction of the distinguished triangle in i) to $\overline{\Sigma_{\mathbf{Q}} \cdot g}$ as the the Morse triangle of $\phi_{g}[-1]\left(\mathbf{Q}^{\bullet}\right)$, with respect to $L$ at $\mathbf{0}$.

Let us return now to the situation of the introduction, where $f: \mathcal{U} \rightarrow \mathbb{C}$ is a complex analytic function whose critical locus, $\Sigma f$, equals $S=\mathcal{U} \cap(\mathbb{C} \times\{\mathbf{0}\})$, i.e., $\operatorname{supp} \phi_{f} \mathfrak{K}_{\mathcal{U}}^{\bullet}=\mathcal{U} \cap(\mathbb{C} \times\{\mathbf{0}\})$. Let $\mathbf{Q}^{\bullet}=\mathfrak{K}_{\mathcal{u}}^{\bullet}[n+1]$. Then, $\mathbf{Q}^{\bullet}$ is perverse, and $\overline{\Sigma_{\mathbf{Q}} f}$ equals the line segment $S$. Thus, the Morse triangle of $\phi_{f}[-1]\left(\mathbf{Q}^{\bullet}\right)$ is a short exact sequence in the category of perverse sheaves on $S$ and, hence, by our discussion in Section 1, is equivalent to a short exact sequence of M-V triangles:

Theorem 2.6. There is a short exact sequence of $M$ - $V$ triangles

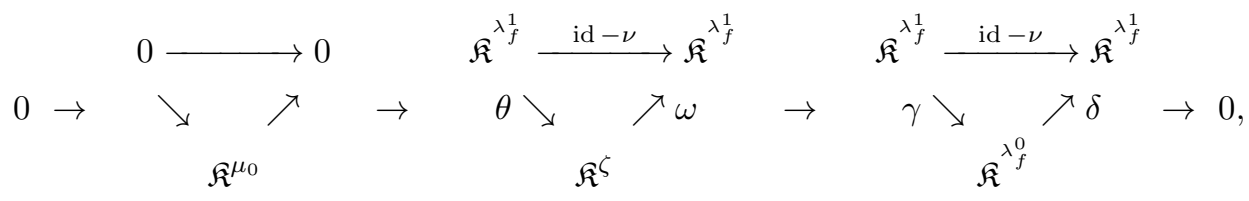

where, as in the introduction and in Section 1, $\nu$ is the internal monodromy, $\lambda_{f}^{1}$ is the 1-dimensional Lê number, $\lambda_{f}^{0}$ is the 0-dimensional Lê number, $\mu_{0}$ is the Milnor number of the isolated critical point (at the origin) of $f_{\left.\right|_{V(L)}}, \zeta:=\mu_{0}+\lambda_{f}^{0}$, and $\theta$ is an injection.

The fact that $\theta$ is an injection follows from property iv) of the Morse modification. We let $\beta$ denote the linear map from $\mathfrak{K}^{\mu_{0}}$ to $\mathfrak{K}^{\zeta}$.

Corollary 2.7. $\operatorname{dim}_{\mathfrak{K}} \widetilde{H}^{n-1}\left(F_{f, \mathbf{0}}\right) \leqslant \lambda_{f}^{1}$, with equality holding if and only if $\operatorname{im} \theta \subseteq \operatorname{im} \beta$.

Proof. As we mentioned in Section $1, \widetilde{H}^{n-1}\left(F_{f, \mathbf{0}}\right) \cong \operatorname{ker} \gamma$; thus, certainly, the inequality holds. In addition, it follows that the equality holds if and only if $\gamma$ is the zero map. As $\theta$ is an injection, $\gamma=0$ is equivalent to $\operatorname{im} \theta \subseteq \operatorname{im} \beta$.

\section{$\S 3$. The Main Theorem}


We return to the setting from the introduction, where we are given a complex analytic function $f:(\mathcal{U}, \mathbf{0}) \rightarrow(\mathbb{C}, 0)$, whose critical locus at the origin is a line. We may select a generic linear form $L$, and consider the one-parameter family of isolated singularities given by $f_{t}:=f_{\left.\right|_{V(L-L(t))}}$, and also consider their corresponding Milnor numbers $\mu_{t}$. Viewing the situation in this from, we see that this $\mu_{0}$ agrees with the $\mu_{0}$ of Theorem 2.6, and that $\lambda_{f}^{1}=\mu_{t}$, for all small $t \neq 0$.

The Milnor monodromy of $f$, induced by letting the value of $f$ travel around a small circle centered at the origin, yields an automorphism, $T$, of the entire short exact sequence of $\mathrm{M}-\mathrm{V}$ triangles in Theorem 2.6; that is, we obtain automorphisms of each vertex of each $\mathrm{M}-\mathrm{V}$ triangle which commute with all the other maps. The main theorem will follow immediately from the existence of these automorphisms, combined with Corollary 2.7 and the following theorem of A'Campo.

Theorem 3.1 (A'Campo, $\left[\mathbf{A}^{\prime} \mathbf{C}\right]$ ). Let $X$ be a complex analytic space, $g: X \rightarrow \mathbb{C}$ a complex analytic function, and $\mathbf{F}^{\bullet}$ a bounded, constructible complex of sheaves of $\mathfrak{K}$-vector spaces on $X$. Let $\mathfrak{m}_{X, \mathbf{p}}$ denote the maximal ideal of $X$ at a point $\mathbf{p} \in V(g)$. If $g \in \mathfrak{m}_{X, \mathbf{p}}^{2}$, then the Lefschetz number of the Milnor monodromy automorphism on the stalk cohomology of $\psi_{g}\left(\mathbf{F}^{\bullet}\right)$ at $\mathbf{p}$ equals 0.

Remark 3.2. In fact, in $\left[\mathbf{A}^{\prime} \mathbf{C}\right], A^{\prime}$ Campo states the above result only in the case where $\mathfrak{K}=\mathbb{C}$. However, one sees easily that his proof is valid for arbitrary fields; by using resolution of singularities (and filtrations and long exact sequences), one is reduced to the case where $g$ is of the form $z_{0}^{\alpha_{0}} z_{1}^{\alpha_{1}} \ldots z_{n}^{\alpha_{n}}$, where the theorem certainly holds over arbitrary fields.

Note that A'Campo's result is a statement concerning $\psi_{g}$, not $\phi_{g}$. We need to discuss the implications for the monodromy automorphism of our short exact sequence of $\mathrm{M}-\mathrm{V}$ triangles in Theorem 2.6 .

The monodromy automorphisms $T_{\mu_{0}}: \mathfrak{K}^{\mu_{0}} \rightarrow \mathfrak{K}^{\mu_{0}}$ and $T_{\lambda_{f}^{1}}: \mathfrak{K}^{\lambda_{f}^{1}} \rightarrow \mathfrak{K}^{\lambda_{f}^{1}}$ are, respectively, the Milnor automorphisms induced on $\widetilde{H}^{n-1}\left(F_{f_{0}, \mathbf{0}} ; \mathfrak{K}\right)$ and $\widetilde{H}^{n-1}\left(F_{f_{t}, \mathbf{0}} ; \mathfrak{K}\right)$ for small $t \neq 0$. As each $f_{t}$ is reduced (in a neighborhood of $\mathbf{0}$ in $\mathcal{U}$ ) and has an isolated critical point at the origin, A'Campo's result implies that the traces are given by $\operatorname{tr}\left(T_{\mu_{0}}\right)=\operatorname{tr}\left(T_{\lambda_{f}^{1}}\right)=(-1)^{n}$.

With all of our preliminary discussion and results, the proof of the main theorem is now simple.

Theorem 3.3. If $\mu_{0}=1+\lambda_{f}^{1}$, then there are strict inequalities

$$
\operatorname{dim}_{\mathfrak{K}} \widetilde{H}^{n-1}\left(F_{f, \mathbf{0}}\right)<\lambda_{f}^{1} \quad \text { and } \quad \operatorname{dim}_{\mathfrak{K}} \widetilde{H}^{n}\left(F_{f, \mathbf{0}}\right)<\lambda_{f}^{0} .
$$

Proof. Recall that in the short exact sequence of M-V triangles in Theorem 2.6, $\widetilde{H}^{n-1}\left(F_{f, \mathbf{0}}\right) \cong \operatorname{ker} \gamma$ and $\widetilde{H}^{n}\left(F_{f, \mathbf{0}}\right) \cong \operatorname{coker} \gamma$. Using the result and notation from Corollary 2.7, we see that the inequalities of the theorem hold, unless $\operatorname{im} \theta \subseteq \operatorname{im} \beta$.

If $\mu_{0}=1+\lambda_{f}^{1}$, then $\operatorname{im} \theta$ and $\operatorname{im} \beta$ are invariant subspaces of the monodromy, whose dimension differs by one, such that the trace of the monodromy on each of the subspaces is $(-1)^{n}$. If $\operatorname{im} \theta \subseteq \operatorname{im} \beta$, 
then the induced monodromy isomorphism on $\operatorname{im} \beta / \operatorname{im} \theta \cong \mathfrak{K}$ would have trace equal to zero; this is impossible.

\section{$\S 4$. Concluding Remarks}

Theorem 3.3 may leave the reader asking several questions:

- Why care about a result which such a restrictive hypothesis?

- Are all of the abstract tools of this paper really necessary to prove Theorem 3.3?

- Does Theorem 3.3 yield the final, general restriction on the cohomology of the Milnor fibre of an affine line singularity?

We address these questions below.

\section{Why care about a result which such a restrictive hypothesis?}

It is notoriously difficult to prove any general statement concerning the cohomology of Milnor fibres of hypersurfaces with non-isolated singularities. It is especially difficult to prove such results where the hypotheses are so easy to calculate effectively, as is the hypothesis of Theorem 3.3.

For example, recall from the introduction that there is an injection

$$
\widetilde{H}^{n-1}\left(F_{f, \mathbf{0}}\right) \hookrightarrow \operatorname{ker}(\mathrm{id}-\nu) .
$$

Thus, if the internal monodromy, $\nu$, is not the identity, then we could again conclude the result of Theorem 3.3, that

$$
\operatorname{dim}_{\mathfrak{K}} \widetilde{H}^{n-1}\left(F_{f, 0}\right)<\lambda_{f}^{1} .
$$

However, it is not so easy, in general, to decide when $\nu$ is the identity. Moreover, it is not true that Theorem 3.3 results from forcing the monodromy to be non-trivial, i.e., it is not true that: if $\mu_{0}=1+\lambda_{f}^{1}$, then $\nu$ is not the identity. Consider $f_{t}:=y^{2}-x^{3}-t^{2} x^{2}$; the reader may verify that the internal monodromy is the identity, $\lambda_{f}^{1}=1, \mu_{0}=2, \lambda_{f}^{0}=5$, and the Milnor fibre at the origin has the homotopy-type of a bouquet of four 2 -spheres.

\section{Are all of the abstract tools of this paper really necessary to prove Theorem 3.3 ?}

This is more difficult to answer. Our proof uses complexes of sheaves to compare the Milnor fibres of $f, f_{0}$, and $f_{t}$ (for $t \neq 0$ ) and their respective monodromies. It may be that there is a more elementary proof. If there is, we do not know of one. Moreover, our proof is really incredibly simple, once one understands the background material. In addition, we believe that the Morse modification and the Morse triangle of Definition 2.5 will prove useful in studying other questions concerning non-isolated hypersurface singularities.

Does Theorem 3.3 yield the final, general restriction on the cohomology of the Milnor fibre of an affine line singularity?

Unfortunately, the answer is: no. This paper was motivated by a result of Siersma in $[\mathbf{S}]$. Translating Sierma's result into the language and notation of this paper, it says: if $\lambda_{f}^{1}=1$ and $\mu_{0} \neq 1$, then 
$\operatorname{dim}_{\mathfrak{K}} \widetilde{H}^{n-1}\left(F_{f, \mathbf{0}}\right)<\lambda_{f}^{1}$, i.e., $\widetilde{H}^{n-1}\left(F_{f, \mathbf{0}}\right)=0$. In terms of M-V triangles, Siersma's result says that, if $\lambda_{f}^{1}=1$ and $\mu_{0} \neq 1$, then the M-V triangle of $\left(\phi_{f} \mathfrak{K}_{\mathcal{U}}^{\bullet}\right)_{\left.\right|_{S}}[n]$ is not the direct sum

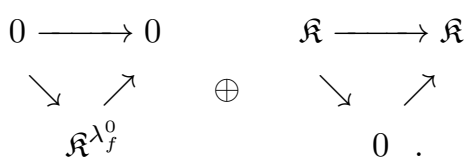

Our result does not imply that of Siersma or, if it does, we do not see how. However, our result and Siersma's have a similar feel, and it is easy to believe that there is one statement and proof that yields both results as special cases of a more general result.

On the other hand, it may be that Theorem 3.3 can, in fact, be used to recover the result of Siersma. The assumption that $\mu_{0}=1+\lambda_{f}^{1}$ is equivalent to the assumption that the generic reduced relative polar curve, $\Gamma_{f, L}^{1}$, has a single smooth component through the origin. In the general case where the polar curve is itself singular, it may be possible to perform an embedded resolution of $\Gamma_{f, L}^{1}$, or of $\Sigma \cup \Gamma_{f, L}^{1}$, and then use Theorem 3.3 to gain information about the Milnor fibre of $f$. Thus far, we have not succeeded with this approach. 


\section{REFERENCES}

[A'C] N. A'Campo, Le nombre de Lefschetz d'une monodromie, Proc. Kon. Ned. Akad. Wet., Series A 76 (1973), $113-118$.

[B] J. Brylinski, Transformations canoniques, Dualité projective, Théorie de Lefschetz, Transformations de Fourier et sommes trigonométriques, Soc. Math. de France, Astérisque 140 (1986).

[D] P. Deligne, Comparaison avec la théorie transcendante, Séminaire de géométrie algébrique du Bois-Marie, SGA 7 II, Springer Lect. Notes 340 (1973).

[K-S] M. Kashiwara and P. Schapira, Sheaves on Manifolds, Grund. der math. Wiss. 292, Springer - Verlag, 1990.

[L] Lê D. T., Calcul du Nombre de Cycles Évanouissants d'une Hypersurface Complexe, Ann. Inst. Fourier, Grenoble 23 (1973), 261-270.

[M-V] R. MacPherson and K. Vilonen, Elementary construction of perverse sheaves, Invent. Math. 84 (1986), $403-435$.

[M1] D. Massey, Hypercohomology of Milnor Fibres, Topology 35 (1996), 969-1003.

[M2] Lê Cycles and Hypersurface Singularities, Lecture Notes in Mathematics, vol. 1615, Springer-Verlag, 1995.

[S] D. Siersma, Isolated Line Singularities, Proc. Symp. Pure Math. 40, Part 2 (1983), 485-496.

David B. Massey, Dept. of Mathematics, Northeastern University, Boston, MA, 02115, USA

E-mail address: DMASSEY@NEU.edu 